\title{
Principles of feature integration in visual perception
}

\author{
WILLIAM PRINZMETAL \\ University of British Columbia, Vancouver, British Columbia V6T 1Y7, Canada
}

\begin{abstract}
Several recent theories of visual information processing have postulated that errors in recognition may result not only from a failure in feature extraction, but also from a failure to correctly join features after they have been correctly extracted. Errors that result from incorrectly integrating features are called conjunction errors. The present study uses conjunction errors to investigate the principles used by the visual system to integrate features. The research tests whether the visual system is more likely to integrate features located close together in visual space (the location principle) or whether the visual system is more likely to integrate features from stimulus items that come from the same perceptual group or object (the perceptual group principle). In four target-detection experiments, stimuli were created so that feature integration by the location principle and feature integration by the perceptual group principle made different predictions for performance. In all of the experiments, the perceptual group principle predicted feature integration even though the distance between stimulus items and retinal eccentricity were strictly controlled.
\end{abstract}

Many information processing theories describe visual pattern recognition in terms of feature abstraction (Bjork \& Murray, 1977; Gibson, 1969; Rumelhart \& Siples, 1974; Selfridge, 1959). According to feature analytic theories, when a subject is presented with a stimulus in a whole report or search experiment, feature detectors are selectively activated by attributes of the stimulus. If enough features are abstracted to discriminate one potential stimulus from another, then the subject can correctly detect or report the stimulus. Incorrect responses are attributed either to accidental activation of inappropriate feature detectors or to not abstracting enough feature information from the display to discriminate between stimuli. An example of the first type of error would be seeing a red letter $X$ when a blue $X$ was presented. An example of the second type of error would be seeing the letter $X$ but not being able to report its color. Both these failures of feature abstraction will be called feature errors (Treisman \& Gelade, 1980).

Recently several theorists have postulated another type of perceptual error that is not related to feature abstraction, but rather to a failure to appropriately join the features that have been correctly abstracted (Treisman, Sykes, \& Gelade, 1977; Wolford, 1975). Two examples of this type of error are as follows.

This research was supported by NSERC of Canada Grant A-7039 to Daniel Kahneman. The author wishes to thank Daniel Kahneman, Anne Treisman, Peter Forster, William Banks, James Steiger, and Robin Mermelstein for their constructive criticism and support, and Janet Souther for her help in conducting this research. Requests for reprints should be sent to William Prinzmetal, Department of Psychology, Princeton University, Princeton, New Jersey 08544.
Suppose colored letters, a red $\mathrm{X}$ and a green $\mathrm{O}$, are briefly presented to a subject. In order to correctly perceive the display, subjects must abstract the features red and green and those features that correspond to the letters $X$ and $O$. Furthermore, subjects must correctly join the features. If the subject abstracts the features of the array but fails to combine them correctly, the subject may report that a green $X$ and a red $O$ were presented. As a second example, consider the stimulus arrays in Figure $1 \mathrm{a}$ or $1 \mathrm{~b}$. If subjects correctly abstract both vertical and horizontal line features, but incorrectly join them, they may perceive an illusory " + ." Errors that result from incorrectly joining features will be called conjunction errors (Treisman \& Gelade, 1980).

Treisman and Schmidt (1981) have shown that conjunction errors are frequent with tachistoscopic exposure in several different tasks. Since this paper assumes that feature integration is a significant process in visual information processing, one of Treisman and Schmidt's experiments will be presented in some detail. In a search task, subjects were briefly presented with three horizontally arranged colored letters, flanked on either side by two black digits. Subjects were required to report first the digits and then whether a predesignated colored target letter was present in the display. There were three types of displays. The first type of displays contained the target. If the target was a red $N$, the stimulus might consist of a red $N$, a green $X$, and a blue $S$. The second type of display was called a conjunction display. In conjunction displays both conjunction errors and feature errors could lead to false alarms. For example, if the target was a red $\mathbf{N}$, the stimulus might consist of a 
green $\mathbf{N}$, a red $\mathrm{X}$, and a blue $\mathrm{S}$. With these stimuli, subjects could make a feature error by misperceiving the green as red, or they could make a conjunction error by inappropriately combining the red of the $X$ with the letter $N$. The third type of display was called a feature display. In feature displays, only feature errors could lead to false alarms. A feature stimulus with a red $\mathbf{N}$ for the target might consist of a green $\mathbf{N}$, a brown $X$, and a blue $S$. In this experiment, there were five possible colors and five possible letters so that if a subject made an error, the probability of a particular feature being in the stimulus array was the same as its not being in the array. The average false-alarm rate was .35 for conjunction displays and only .22 for feature displays (the hit rate was .68). In other words, if it were possible to misjoin features in the stimulus in a way that resulted in a target, subjects would be one-third more likely to make a false alarm than they would be if such a conjunction were not possible. Treisman and Schmidt have also found evidence for conjunction errors in a whole-report task, as have Wolford and Shum (1980). Finally, Treisman and Schmidt have shown, in a simultaneous matching task, that a false-alarm rate that is higher with conjunction stimuli than with feature stimuli is not the result of merely matching the features in the display with the features of the target. In this experiment, subjects were briefly presented with five colored letters and had to determine whether any two stimulus items had the same shape and color. Treisman and Schmidt found that the number of possible conjunction errors that would lead to a false positive for a particular stimulus predicted performance independently of the total number of matching features in the stimulus.

The purpose of the present study was to use conjunction errors to investigate principles that could predict which features of a stimulus display would be integrated by the visual system. Specifically, this research sought to determine whether the visual system was more likely to join features from stimulus items that were located close together in visual space (the location principle) or whether it was more likely to join features from stimulus items that formed the same perceptual group or object (the perceptual group principle).

These possible principles of feature integration, "the location principle" and "the perceptual group principle," follow from very different kinds of theories of feature integration and make unique predictions. Theories consistent with the location principle use only location information for feature integration. The principle is very simple: features from the same or neighboring locations in visual space are likely to be joined. Theories consistent with the location principle would predict that the probability of a conjunction error (such as switching the color in two adjacent letters) would increase as the distance between items decreased. Wolford's (1975) feature perturbation theory is an example of a theory that is consistent with the location principle. According to Wolford, features are abstracted with location information. The location information decays with time and features perturbate according to a random walk process. There is, therefore, a greater probability that features will perturbate a short distance than that they will a long distance. In addition to a distance effect, Wolford's theory also predicts that the greater the eccentricity of the stimulus item in the visual field, the greater the probability of feature perturbations. Finally, Wolford's theory predicts that features are more likely to perturbate in a central direction than in a peripheral direction. These last two predictions do not, of course, follow strictly from the location principle.

Theories consistent with the perceptual group principle use information about the perceptual organization or structure of the display for feature integration. Perceptual organization is determined by information about similarity, proximity, goodness of form, and so on. The general principle is this: Features from the same perceptual group or object are likely to be joined. Theories consistent with this principle predict that illusory conjunctions are more likely to form between items in the same perceptual group than between items in different perceptual groups. The term "perceptual group" is used in this paper to describe the results of parsing the stimulus array in accordance with the Gestalt principles of organization. Fox's continuity theory (Fox, 1978) is one example of a theory that is consistent with the perceptual group principle. According to Fox, early in visual information processing, "object-tokens" are created by the visual system on the basis of similarity, texture, symmetry, and so on. As more information becomes available, or as more features are abstracted, descriptions are added to the object-tokens. Features added to the same object-token are, in the terminology used here, integrated. It should be emphasized that Fox's theory is just one of a class of theories consistent with the principle that features from the same perceptual group are likely to be joined. Likewise, Wolford's theory is just one theory in a class of theories that postulates that feature integration is mediated only by item location.

The visual world is so constructed that the perceptual group principle and the location principle generally lead to the same prediction. It is generally true that features that are abstracted from the same region of visual space belong to the same object or perceptual unit. For example, both principles predict more conjunction errors between adjacent letters than between nonadjacent letters. The location principle would make this prediction because the distance between adjacent items would be less than the dis- 
tance between nonadjacent items. The perceptual group principle would make this same prediction because adjacent items are more likely to form a single perceptual unit by proximity grouping. Both Snyder (1972) and Wolford and Shum (1980) have found more conjunction errors with adjacent than with nonadjacent stimuli. Snyder, for example, briefly presented 12 letters in a circle. One of the letters was red, and subjects were to report the identity and location of the red letter. When the subjects reported the letter incorrectly (but the location correctly), they were more likely to report a letter adjacent to the target than any other letter.

In the experiments reported in this paper, a situation was created in which theories consistent with the location principle and theories consistent with the perceptual group principle make different predictions for feature integration. Specifically, I looked for an effect of perceptual organization on feature integration while controlling for the effects of item location. To understand how this was accomplished, consider the eight stimuli in Figures 1 and 2. As in all the figures in this paper, the fixation point is in the exact center of each panel. These figures represent the stimulus conditions in Experiment 1. On each trial, a subject was briefly presented with a stimulus like one of those in Figures 1 and 2, and the subject had to respond "yes" or "no," depending on whether the stimulus contained a plus. The correct response for any of the four stimuli in Figure 2 is, of course, "yes." The correct response for any of the stimuli in Figure 1 is "no." The interesting responses in this experiment are the false alarms that occur with stimuli like those in Figure 1. Figures $1 \mathrm{a}$ and $1 \mathrm{~b}$ are samples of conjunction stimuli. If vertical and horizontal

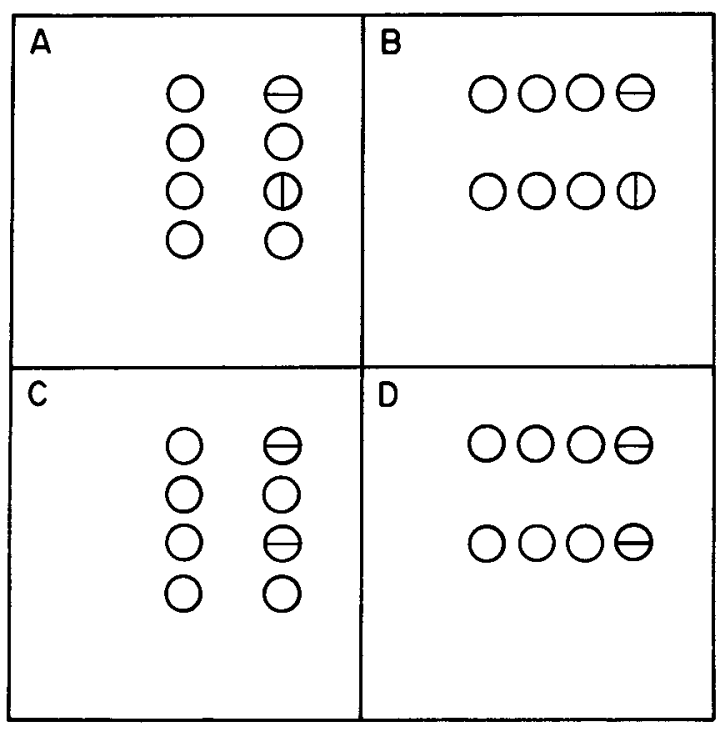

Figure 1. Examples of the target-absent stimuli in Experiment 1. The fixation point is the exact center of each panel.

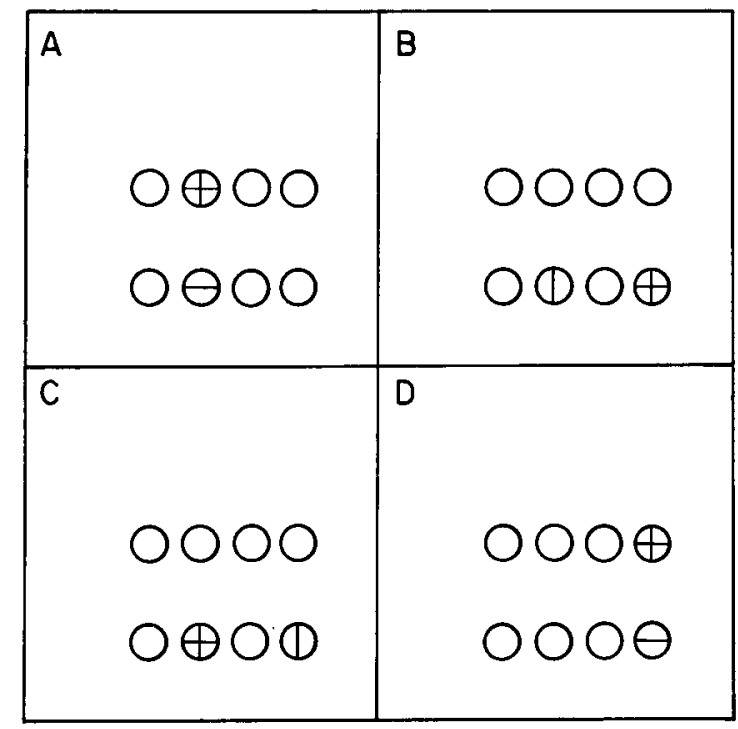

Figure 2. Examples of the target-present stimuli in Experiment 1.

line features in these displays were correctly abstracted, but incorrectly joined, subjects might perceive an illusory plus and make a false alarm. Figures $1 \mathrm{c}$ and $1 \mathrm{~d}$ represent samples of feature stimuli. These stimuli contained either two vertical or two horizontal lines. (Samples of horizontal line segments are shown in Figures $1 \mathrm{c}$ and $1 \mathrm{~d}$.) Incorrectly joining these features would not produce an illusory plus. The first prediction in this experiment is that there would be more false alarms with stimuli like Figures $1 \mathrm{a}$ and $1 \mathrm{~b}$ than with stimuli like Figures $1 \mathrm{c}$ and 1d. This result would extend the findings of Treisman and Schmidt and would provide evidence that at least some of the false alarms in this experiment were due to illusory conjunctions. The predictions that are critical to distinguishing feature integration by the location principle vs. feature integration by the perceptual group principle concern a comparison of false alarms in Figures $1 \mathrm{a}$ and $1 \mathrm{~b}$. The vertical and horizontal lines are in the same physical location in these two displays, but in Figure 1a they are in the same perceptual unit or group, while in Figure $1 \mathrm{~b}$ they are in different perceptual groups. If the location principle is correct, there should be no difference in false-alarm rate in these two conditions because distance and retinal eccentricity are the same in both cases. If the perceptual group principle is correct, then there should be more false alarms with stimuli like the one in Figure 1a because both horizontal and vertical lines are in the same group.

\section{EXPERIMENT 1}

\section{Method}

Procedure. Subjects were briefly presented with a stimulus like those in Figures 1 and 2 and had to respond verbally "yes" or 
"no" as to whether the stimulus contained a plus. The subjects were tested individually with a two-field Cambridge tachistoscope. The stimulus items were preceded and followed by a visual noise mask made up of black dots. The dot in the center of the mask was enlarged slightly to serve as a fixation point. Just before each trial, the experimenter said "ready" and then immediately initiated the trial. The subjects responded by saying "yes" if they thought that the display contained a plus and "no" if they thought that the display did not contain a plus. The subjects were given as much time as they wanted to make their decisions, and reaction time was not measured. Following a subject's response, the next trial began.

Each subject participated in one session that lasted about $1 \mathrm{~h}$. The session began with the experimenter showing the subject many of the stimulus cards outside the tachistoscope and explaining the task. The subjects were told that "approximately half the stimuli contained pluses," but, in actuality, only one-third contained pluses (see below). The subjects were given 50 practice trials. During practice, the exposure duration was lowered until the subjects responded correctly on approximately $85 \%$ of the trials. This exposure duration was used for the first block of data trials. Data were then collected over six blocks of 96 trials each, with a short rest halfway through each block. At the end of each block, the subjects were told their percent correct on the block and the exposure duration was increased or decreased to maintain approximately $85 \%$ correct responding. The mean exposure duration was $80.2 \mathrm{msec}$ (range 130 to $30 \mathrm{msec}$ ). The 96 stimuli were presented in a different random order on each block.

Stimuli. Thirty-two of the 96 stimuli contained a target plus and 64 did not contain a plus. Thirty-two of the 64 target-absent stimuli resulted from all eight $90-$ deg rotations and reflections of the stimuli shown in Figure 1, and there were 32 displays that were identical to these except for the substitution of vertical line segments for horizontal line segments, and vice versa.

The circles of the 32 stimuli that contained pluses were located in the same eight rotations and reflections as the target-absent stimuli. For a particular rotation/reflection of the two lines of circles, the plus could be located in one of four relative positions. Three of the positions corresponded to the same positions as could be occupied by a vertical or horizontal line segment in the targetabsent stimuli, and the fourth position was in the circle nearest the fixation point (see Figure 2). Each of the target-present stimuli contained a plus and also an additional line segment. This additional line segment was vertical on half the stimuli and in the same line of circles as the target plus on half the stimuli. The reason for including this extra line segment was to prevent subjects from responding to the stimuli on the basis of whether they saw one "black thing" in the array or two "black things" in the array (see below).

The circles were drawn with blue felt-tipped pens (Wik Stik turquoise blue), and the vertical/horizontal lines were drawn with a black felt-tipped pen on a white background. In this and all the experiments reported in this paper, the circles were $3 / 8$ in. in diameter (subtending a visual angle of $1.432 \mathrm{deg}$ ). The distance between the centers of adjacent circles was $1 / 2$ in., and the distance between the centers of the circles opposite to one another was 1 in. The masking field was made with a sheet of Letratone \#LT 113. The masking field consisted of a regular pattern of small black dots, approximately $1 / 16 \mathrm{in}$. in diameter. The stimulus displays were viewed binocularly from a distance of 15 in.

Subjects. Twelve subjects (seven female), ages 20 to 27 years, were recruited from the subject pool at the University of British Columbia. All subjects had normal or corrected-to-normal vision and were paid $\$ 3$.

\section{Results and Discussion}

The proportion of false alarms for Experiment 1 is presented in Table 1 . The results are clearly con-
Table 1

False Alarms in Experiment 1

\begin{tabular}{lccc}
\hline & $\begin{array}{c}\text { Same } \\
\text { Group }\end{array}$ & $\begin{array}{c}\text { Different } \\
\text { Group }\end{array}$ & Mean \\
\hline Conjunction & .22 & .15 & .185 \\
Feature & .07 & .05 & .06 \\
Mean & .145 & .10 & \\
\hline
\end{tabular}

sistent with feature integration by the perceptual group principle. The proportion of false alarms was significantly greater with the conjunction than with the feature stimuli, .185 vs. $.06[F(1,11)=42.05, \mathrm{p}<.001]$ There were more false alarms when the two line segments were in the same perceptual group than when they were in different perceptual groups, .145 vs. .10 $[F(1,11)=8.07, p<.025]$. Finally, there was a significant interaction between these two variables $[F(1,11)$ $=6.11, p<.05]$. A simple effects test showed that there was a significant difference between having the line segments in the same vs. different groups for conjunction displays $[\mathrm{t}(11)=3.11, \mathrm{p}<.005]$, but not for feature displays $[t(11)=.98]$. The prediction of theories consistent with the perceptual group principle was that there would be more false alarms when a vertical and a horizontal line were in the same group than when they were in different groups (Figure la vs. Figure (b). However, there was no reason to make this prediction for feature stimuli (Figure 1c vs. Figure 1d), and no difference was found in this experiment.

The finding of a difference between conjunction stimuli and feature stimuli in false-alarm rate was a necessary prerequisite for concluding that the effects of perceptual organization in this experiment were the result of feature integration and not some other process. The finding that there were more false alarms with conjunction than with feature displays is one indication that at least some of the false alarms were due to incorrectly joining vertical and horizontal line features. It could be argued that more false alarms with conjunction displays simply indicate that subjects are responding by counting the number of unique target features that are in the stimulus. A conjunction stimulus would have at least two target features (a vertical and a horizontal line feature), while a feature stimulus might have only one target feature (a vertical or a horizontal line feature). A feature-counting model could explain the difference in false-alarm rate between conjunction and feature stimuli without postulating that any features were integrated or that there were illusory pluses. However, a feature-counting model cannot explain the main results of the experiment-the effect of the features being in the same or different perceptual groups. Furthermore, it is clear from subjects' spontaneous comments that subjects did, on occasion, "see" illusory pluses. All 
the subjects were told that there was at most one plus on each stimulus and that when there wasn't a plus there were at most two lines. Five of the subjects spontaneously commented that they sometimes saw more than one plus or more than two lines. A typical comment was for the subject to ask, after the first block of trials, whether there were some stimulus cards with more than one target plus.

The analysis of performance on target-present trials (i.e., hit rate) is important because it also supports the claim that the effects of perceptual organization are due to failures in feature integration and not to a failure in feature extraction. The hit rates were analyzed by the relative position of the target in the display. The target could be located at the fixation point (Figure $2 \mathrm{a}$ ), in the circle farthest from the fixation point (Figure 2b), in the circle opposite to the fixation point (Figure 2c), or in the circle at the end of the line of circles that included the fixation point (Figure 2d). The hit rates for the pluses in these four positions were $.995, .969, .976$, and .896 for displays like those in Figures 2a, 2c, 2d, and 2b, respectively. These hit rates are ordered according to retinal eccentricity of the pluses (with $\mathrm{c}$ and $\mathrm{d}$ at the same retinal eccentricity) and are reliably different $[F(3,33)=11.78$, $\mathrm{p}<.001$ ]. A perhaps subtle, but very important, finding is that when the pluses were at the same retinal eccentricity (Figures 2c and 2d), hit rates were not significantly different $[t(11)=.08]$. To understand why this finding is important, consider Figures 1a and $1 \mathrm{~b}$. The claim made was that there were more false alarms with stimuli like Figure 1a than like Figure $1 \mathrm{~b}$ because of inappropriate feature integration, not because of a failure in feature extraction. This conclusion is justified only if it is equally easy to extract feature information in these two stimuli. Suppose, for example, it were harder to see (extract information from) the vertical line in Figure 1a because it is flanked on two sides. If this were the case, it might be argued that the higher false-alarm rate for the stimulus in Figure la was due to the fact that it was harder to see the vertical line in this stimulus and therefore subjects had to guess more often. The hit data show, however, that it was as easy to see something (the plus) in the position of the vertical line in Figure 1a as it was in Figure 1b. This discussion highlights the fact that, in experiments dealing with feature integration, it is very important to equate the ease of feature extraction across conditions.

Hit rates were further analyzed to determine if finding more false alarms with the same group stimuli than with different group stimuli was due to a response bias. Response bias refers to any factor, extrinsic to the encoding process, that would cause subjects to be more likely to respond "yes" to one class of stimuli and "no" to another class of stimuli. Suppose subjects adopted the strategy that when they were unsure of whether the stimulus contained a plus, it would be better to guess "yes" if the black lines were in the same group and "no" if they were in different groups. It is important to note that there was no formal reason to adopt such a strategy. However, such an irrational strategy could cause the main effect of same vs. different group with the false alarms, and it is important to determine whether subjects adopted this strategy (cf. Santee \& Egeth, 1980). If subjects were more likely to respond "yes" when the black lines were in the same group and "no" when they were in different groups, then there should be a higher hit rate when the plus and extra line were in the same group (e.g., Figures $2 b, 2 c$ ) than when they were in different groups (e.g., Figure 2d). This analysis of hits included only those target-present stimuli with the plus and the extra line in exactly the same positions as the vertical and horizontal lines in the targetabsent stimuli. Hence, stimuli such as those in Figure $2 \mathrm{a}$ were not included because there were no targetabsent stimuli with a line at the fixation point. In this analysis, the same and different group target-present stimuli were exactly the same as the same and different group target-absent stimuli except for the presence of an additional line that created a plus. The hit rates for the lines being in the same vs. different group were not significantly different [.934 vs. .922; $\mathrm{t}(11)=.83$ ]. Although it is difficult to prove the null hypothesis, there does not seem to be much support for a response bias explanation of the effect of perceptual organization (same vs. different group). A much more powerful test of this response bias hypothesis is presented in Experiment 4.

The results of Experiment 1 show that in this task subjects do make errors of feature integration. Furthermore, when subjects make these conjunction errors they are more likely to join features from the same perceptual group than from different perceptual groups, even when the distance between items and retinal eccentricity are controlled. The purpose of the second experiment was to replicate Experiment 1 to make sure that the results were not an artifact of the particular display organization used, but represented a general principle of feature integration.

\section{EXPERIMENT 2}

\section{Method}

The design and procedure in Experiment 2 were exactly the same as in Experiment 1. Only the stimulus organization was different. Samples of the target-absent stimuli are shown in Figure 3. Figures $3 \mathrm{a}$ and $3 \mathrm{~b}$ are conjunction stimuli; Figures $3 \mathrm{c}$ and $3 \mathrm{~d}$ are feature stimuli. In Figures $3 a$ and $3 c$, the vertical and/or horizontal lines are in the same perceptual group, whereas in Figures $3 \mathrm{~b}$ and 3d they are in different perceptual groups. As in Experiment 1, the distance between the circles with lines in them was identical for same and for different group displays. To control for retinal eccentricity, the distance between the centers of the two circles containing lines in them and the fixation point is exactly matched 


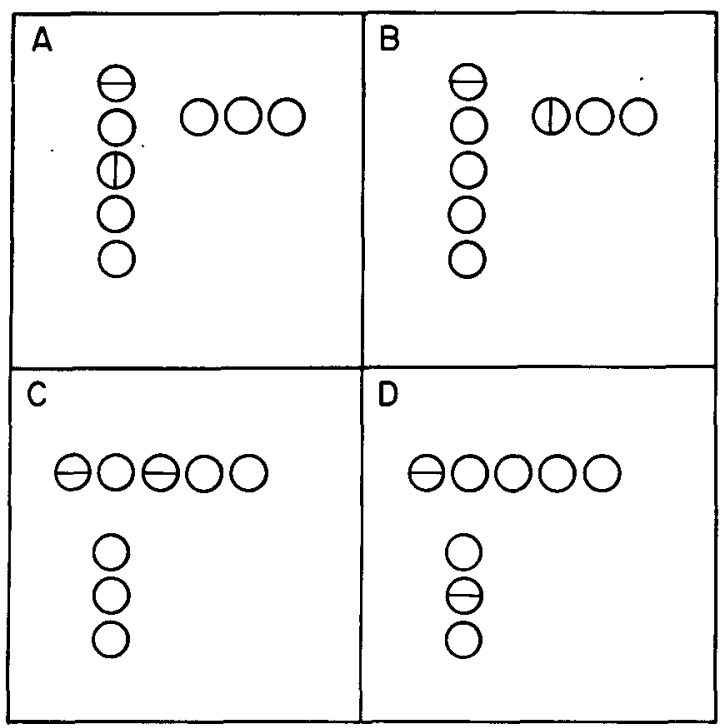

Figure 3. Examples of the target-absent stimuli in Experiment 2.

for same vs. different group displays. The complete set of 64 targetabsent stimuli was generated from Figure 3 in the same manner as it was generated from Figure 1 in Experiment 1. Similarly, the 32 target-present displays (no examples shown) were generated as in Experiment 1. The target plus could be located in the same three circles as the circles with vertical or horizontal lines in them in Figure 3 . In 16 target-present stimuli, the plus was in the corner position (two for each rotation/reflection), and in 8 stimuli the plus was located in one of the other two positions. These two circles are equidistant from the fixation point. An extra vertical or horizontal line was drawn in the target-present stimuli, as in Experiment 1 . In half of the target-present stimuli, the target plus and extra line were in the same group, and in half they were in different perceptual groups. As in Experiment 1, the circles were drawn in blue, the lines in black. The distance between the centers of adjacent circles was $7 / 16$ in., and the distance from the fixation point to the centers of the two nearest circles was $3 / 4$ in. The stimuli were viewed from a distance of $15 \mathrm{in}$. as they were in all the experiments reported in this paper. The average exposure duration was $106.5 \mathrm{msec}$ (range 150 to $50 \mathrm{msec}$ ). Twelve subjects, four female, were recruited as in Experiment 1.

\section{Results and Discussion}

The proportion of false alarms for Experiment 2 is presented in Table 2 . The results are very similar to those in Experiment 1 and support theories consistent with the perceptual group principle. The proportion of false alarms was significantly greater with conjunction than with feature arrays, .22 vs. .095 $[F(1,11)=77.60, p<.001]$. There were significantly

Table 2

False Alarms in Experiment 2

\begin{tabular}{lccc}
\hline & $\begin{array}{c}\text { Same } \\
\text { Group }\end{array}$ & $\begin{array}{c}\text { Different } \\
\text { Group }\end{array}$ & Mean \\
\hline Conjunction & .26 & .18 & .22 \\
Feature & .11 & .08 & .095 \\
Mean & .185 & .13 & \\
\hline
\end{tabular}

more false alarms when the line segments were in the same perceptual group than when they were in different perceptual groups, . 185 vs. .13 $[\mathrm{F}(1,11)=18.97$, $p<.005]$. As in Experiment 1, the difference in falsealarm rate for same vs. different group was greater for conjunction stimuli than for feature stimuli. However, unlike the first experiment, this interaction was only marginally reliable $[F(1,11)=3.99, p=.07]$. The difference between same and different group stimuli was reliable for both conjunction and feature displays $[\mathrm{t}(11)=3.46, \mathrm{p}<.005$ and $\mathrm{t}(11)=2.36$, $\mathrm{p}<.05$, for conjunction and feature displays, respectively]. The results of the false-alarm analysis replicate Experiment 1 in every way except for the small and unpredicted difference between same and different groups for feature stimuli. The effect of same vs. different group on feature stimuli will be addressed in the discussion of Experiment 3.

Hit rate did not vary as a function of relative position in the configuration of the target $(F<1.0)$. When the target was in the corner position (i.e., position of horizontal line in Figure 3a), the hit rate was .966. When the target was in the position in the middle of the group of five circles (i.e., position of vertical line in Figure 3a), the hit rate was .964. Finally, when the target was in the group of three circles (i.e., position of the vertical line in Figure 3b), the hit rate was 969. As in Experiment 1, hit rates did not vary as a function of whether the lines were in the same vs. different groups, .972 vs. $.969[\mathrm{t}(11)=.68]$.

In Experiments 1 and 2, the perceptual group principle predicted more false alarms when the target features were in the same perceptual group than when the target features were in different perceptual groups. The location principle predicted no difference between these conditions. The results clearly support the perceptual group principle. The purpose of the third experiment was to create a situation in which feature integration by the perceptual group principle and feature integration by the location principle make opposite predictions.

\section{EXPERIMENT 3}

Experiment 3 was very similar to Experiment 1 except that in this experiment target line features in the same perceptual group were actually further apart than target line features in different perceptual groups. Samples of the target absent stimuli are shown in Figure 4. The distance between the centers of circles with lines in them subtended $5 \mathrm{deg}$ of visual angle (1-5/16 in.) when the lines were in the same perceptual group (Figures $4 \mathrm{a}$ and $4 \mathrm{c}$ ), but only $4 \mathrm{deg}$ of visual angle (1-1/16 in.) when the lines were in different perceptual groups (Figures $4 \mathrm{~b}$ and $4 \mathrm{~d}$ ). Theories consistent with the location principle would now predict fewer false alarms for stimuli like Figures $4 a$ and 


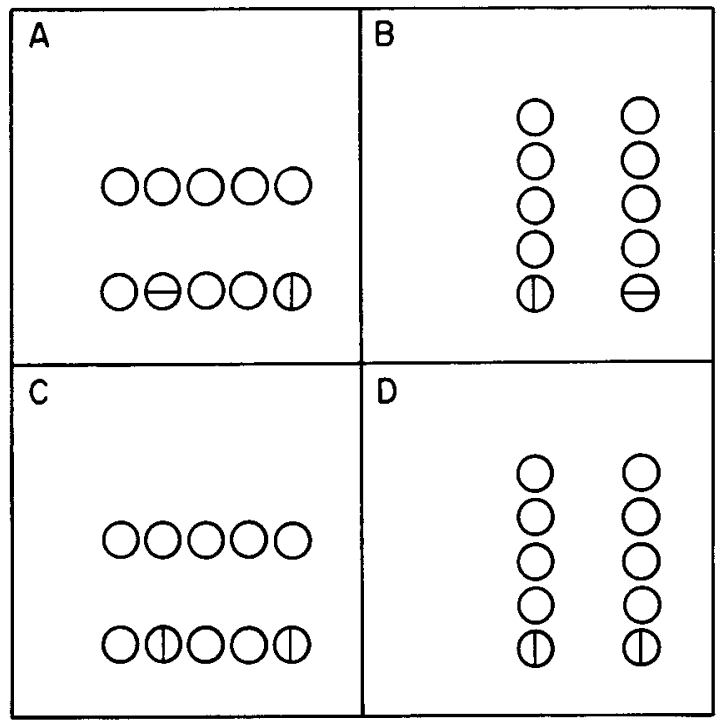

Figure 4. Examples of the target-absent stimuli in Experiment 3.

$4 \mathrm{c}$ because the line segments in these stimuli are located farther apart than those in Figures $4 \mathrm{~b}$ and $4 \mathrm{~d}$. Theories consistent with the perceptual group principle would make the opposite prediction.

\section{Method}

The method, design, and procedure in this experiment were exactly the same as in Experiment 1, except that both the line segments and circles were drawn in black. The average exposure duration was $101.9 \mathrm{msec}$ (range 150 to $30 \mathrm{msec}$ ). Twelve subjects, four female, were recruited as before.

\section{Results and Discussion}

The proportion of false alarms for Experiment 3 are presented in Table 3. As in the previous two experiments, there were significantly more false alarms with conjunction stimuli than with feature stimuli, .24 vs. $.105[F(1,11)=20.30, p<.001]$. Furthermore, there were significantly more false alarms when the vertical or horizontal lines were in the same perceptual group than when they were in different perceptual groups, .19 vs. $.155[F(1,11)=8.15$, $p<$ $.025]$. This result is clearly in line with feature integration by the perceptual group principle. Features from stimulus items that belong to the same perceptual group were more likely to form an illusory conjunction even though they were located farther apart

Table 3

False Alarms in Experiment 3

\begin{tabular}{lccc}
\hline & $\begin{array}{c}\text { Same } \\
\text { Group }\end{array}$ & $\begin{array}{c}\text { Different } \\
\text { Group }\end{array}$ & Mean \\
\hline Conjunction & .26 & .22 & .24 \\
Feature & .12 & .09 & .105 \\
Mean & .19 & .155 & \\
\hline
\end{tabular}

than features from stimulus items belonging to different perceptual groups.

Unlike the results of the first two experiments, the interaction between same vs. different group and feature vs. conjunction stimuli did not approach significance $(F<1.0)$. The difference in false-alarm rate for same vs. different group was reliable for conjunction displays $[\mathrm{t}(11)=2.19, \mathrm{p}<.05]$, and it was also reliable for feature displays $[t(11)=2.06, p<.05]$. In retrospect, it seems that the effect of perceptual organization on feature stimuli increased from Experiment 1 to Experiment 3, while the effect on conjunction stimuli decreased slightly. The following explanation can, I believe, account for these results. The vertical and horizontal lines were probably easiest to see in the first experiment and hardest to see in the third experiment. In the first two experiments, the black vertical and horizontal lines stood out clearly on the blue circles. In the third experiment, the circles and line segments were both black. There were fewer stimulus items in the first experiment, and they were spaced farther apart than in the second experiment. All these factors probably made it easiest to correctly extract feature information in the first experiment and hardest in the third experiment. Consider a feature stimulus with two horizontal lines. If one of these horizontal lines is encoded as a vertical line, then the stimulus is functionally like a conjunction stimulus. If the two line segments are from the same perceptual group, then they would be more likely to form an illusory plus than if they were in different groups. Similarly, miscoding one horizontal or vertical line in a conjunction stimulus would cause that stimulus to be functionally equivalent to a feature stimulus. I am suggesting that such miscoding happened most often in the third experiment and least often in the first experiment. This would explain why feature stimuli became more like conjunction stimuli, and vice versa, in the results from the first experiment to the third experiment. This explanation fits the results well, but it is post hoc.

The analysis of hit rates replicated the results of Experiment 1 . The hit rates significantly varied with retinal eccentricity $[F(3,33)=6.00, p<.005]$. When the target was in the position nearest the fixation point, the hit rate was .991 , and when the target was in the position farthest from fixation, the hit rate was .908. When the target was in the intermediate positions (these being equidistant from the fixation point), the hit rates were .969 and .972 . These latter two hit rates are not significantly different $[t(11)=$ .69]. Hit rates were analyzed, as in the previous experiments, for those stimuli with lines in the same vs. different perceptual groups. There were slightly more hits when the lines were in the same vs. different groups (.962 vs. .934). This effect failed to reach sig- 
nificance $[t(11)=1.57]$, and only 6 of the 12 subjects showed this effect.

\section{EXPERIMENT 4}

In the first three experiments, perceptual organization was manipulated by the geometric relationships between the circles. In this final experiment, item similarity was used to manipulate perceptual organization. In order to create the desired perceptual organization, the stimulus circles were colored in with one of two colored transparent marker pens of the type used to highlight text. Consider the stimuli in Figure 5a. Without the color, the circle located where the two lines of circles meet could group with either the three horizontally aligned circles or with the three vertically aligned circles. When the circles were colored with a transparent color marker, this corner circle effectively formed a perceptual group with either the horizontally oriented circles (Figures $5 \mathrm{a}$ and $5 \mathrm{c}$ ) or the vertically oriented circles (Figures $5 \mathrm{~b}$ and $5 \mathrm{~d}$ ). This manipulation caused the line segments to be in the same perceptual group (Figures $5 \mathrm{a}$ and $5 \mathrm{c}$ ) or in different perceptual groups (Figures $5 b$ and $5 d$ ). Unfortunately, it is impossible to demonstrate exactly what the stimulus displays looked like in a black and white figure. However, circles that were colored alike did phenomenally form strong perceptual groups, and the colors apparently did not make it much harder, if at all, to perceive the vertical and horizontal lines.

\section{Method}

The 64 target-absent stimuli can be generated from Figure 5 . There were eight rotations and reflections of these stimuli, vertical

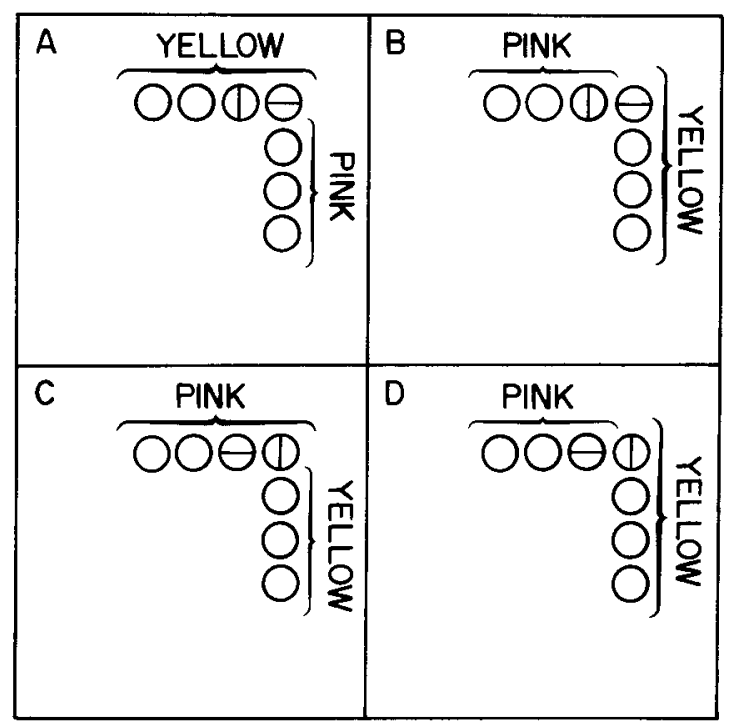

Figure 5. Examples of the target-absent stimuli in Experiment 4. The centers of the circles were colored with a transparent marker, as indicated by the brackets. lines were substituted for horizontal lines and vice versa, and pink was substituted for yellow and vice versa. All the target-absent stimuli were conjunction stimuli. For each rotation and reflection, there were four target-present stimuli, two with the target on the corner position and two with the target in a circle next to the corner position. On half of the target-present stimuli, the target was in a pink circle, and on half $t$ was in a yellow circle. The extra line in the target-present stimuli was either in the corner position or in one of the circles adjacent to the corner circle. The extra line was in a circle of the same color as the target on half the stimuli and was vertical on half the stimuli.

The distance between the centers of adjacent circles was $7 / 16$ in., and the distance from the fixation point to the center of the two circles next to the corner circle was 1 in. The circles and lines were drawn in black. The centers of the circles were colored in either yellow or pink with a Staedtler Top-Marker pen.

The procedure was exactly the same as in the previous experiments. The average exposure was $112.8 \mathrm{msec}(150$ to $60 \mathrm{msec})$. Twelve subjects, five female, were recruited as before.

\section{Results and Discussion}

The results in this experiment are very easy to relate. The probability of making a false alarm was greater when the vertical and horizontal lines were in the same group (i.e., circles with the same color) than when they were in different groups (i.e., circles with different colors), .235 vs. .191. This difference was reliable $[F(1,11)=9.09, p<.025]$. Among the same group stimuli, the false-alarm rate was the same when the lines were in pink circles $(.236)$ and when they were in yellow circles $(.234)(\mathrm{F}<1.0)$.

Hit rate did not vary significantly as a function of the target position. The hit rates when the target was in the corner and off-corner positions were .925 and .943 for corner and off-corner positions, respectively $(F<1.0)$. It may have been slightly easier to see the targets in the pink circles than in the yellow circles $[.921$ vs. $.947 ; F(1,11)=4.23, p=.064]$.

Target-present stimuli were further analyzed to compare hit rates when the target and the extra line in the same perceptual group vs. different perceptual groups. A response bias explanation of the difference in false alarms between same and different group stimuli would predict more hits when the lines were in the same group than when they were in different groups. Hit rates for same vs. different group stimuli were .933 vs. .940 for same and different group stimuli, respectively. These were not reliably different $[t(11)$ $=.49$ ]. To obtain a statistically more powerful test of this response bias hypothesis, all four experiments were pooled in an ANOVA on hit rates. Factors were the plus and extra line being in the same vs. different groups, experiments, and subjects nested in experiment. Hit rate did not vary significantly as a function of the lines being in the same or different groups $[F(1,44)=1.73]$. Only 22 of the 48 subjects had higher hit rates when the lines were in the same vs. different groups. An analysis on $\mathrm{d}^{\prime}$, using the same design as with hits, also failed to provide evidence for the response bias hypothesis. The $\mathrm{d}^{\prime}$ scores were derived 
from the same and different perceptual group hit rates, as above, and from the false-alarm rates from the same and different group conjunction displays. Unlike the analysis of hits, there was a significant effect of perceptual group on $d^{\prime}[F(1,44)=6.24, p<$ $.025]$. In both the analysis of hits and the analysis of $d^{\prime}$, perceptual group did not significantly interact with experiment. There are, perhaps, several reasons why analysis of $d^{\prime}$ may not be appropriate in the pressent experiments. ${ }^{1}$ Nevertheless, the analysis of hits and $\mathrm{d}^{\prime}$ taken together provide consistent evidence that the effect of same vs. different groups on false alarms was not caused by a response bias.

In this experiment, as in Experiment 1, the vertical and horizontal lines were in exactly the same physical position when they were in the same or different perceptual groups. Feature integration by the location principle does not predict a difference in false alarms as a function of item similarity. Feature integration by the perceptual group principle does predict that item similarity will affect feature integration, because similar items tend to group together. Such an effect was found.

\section{GENERAL DISCUSSION}

The results show that perceptual organization affects feature integration. Features from stimulus items that belong to the same perceptual group are more likely to be integrated than are features from stimulus items that belong to different perceptual groups, even when the distance between items and retinal eccentricity are strictly controlled. There is a good functional reason why this general principle should be true. It is reasonable to assume that the unit formation processes that cause perceptual grouping are the same processes that divide the visual world into objects. The visual world would be chaos if features from different objects were free to combine. There is a large class of theories that could account for the perceptual group principle. Several examples of theories consistent with this principle are presented below. Before discussing these theories, however, the interpretation of this research and the meaning of the term "feature" should be clarified.

It should be made clear that this research does not rule out the possibility that item location, independent of perceptual organization, also has an effect on feature integration. In Experiment 3, the distance between items and perceptual grouping were pitted against each other to see which would affect feature integration. Perceptual organization predicted the resulting feature integration, and the distance between items did not. However, it may be that perceptual organization was manipulated in a relatively powerful way and the distance between items in a relatively weak way. The research reported here shows that when the distance between items and retinal eccentricity are controlled, the perceptual group principle predicts feature integration. To show that the location principle predicts feature integration independently of perceptual organization, one would have to control for the effects of perceptual organization and look for an effect of item location. Since the effects of perceptual organization are ubiquitous, this may be difficult to do. For example, since stimulus items that are located close together are more likely to group together by proximity grouping, it may be difficult to separate the effects of proximity grouping and the distance between stimulus items.

The term "feature" has several different meanings in psychology, and therefore it is important to understand what is meant by a feature in this paper. For example, features are thought of as (1) the basic, irreducible units of perception, (2) the output of specific neural units, or (3) the independent psychological codes used by the visual system for recognition of a particular set of stimuli. These definitions are not necessarily mutually exclusive, of course. A psychological code may or may not correspond to the output of a specific neural unit. The term "feature" in this paper, however, is restricted to the independent psychological codes which combine to mediate the recognition of, for example, a plus. It has been assumed that two of the psychological features of the target "plus" in this research are "horizontal line segment" and "vertical line segment." Part of the justification for the assumption is that the experiments worked, that is, that there were more conjunction errors than feature errors. If this were the only operational definition of an independent feature, the reasoning in this experiment would be circular. Fortunately, there are many other operational definitions that could justify horizontal and vertical lines as independent, separable features (Garner, 1974; Treisman \& Gelade, 1980). The choice of features need not, in principle, be circular.

Theories of feature integration will have to account for the influence of perceptual organization. Three theories, chosen for their diversity, are presented here. Fox's continuity theory (Fox, 1978) was briefly mentioned in the introduction. The theory is based, to some extent, on the computer vision model of Marr (Marr, 1976). Early visual information processing "object-tokens" are assigned to the visual input on the basis of similarity, texture, symmetry, proximity, and so on. Object-tokens are purely internal information processing symbols that keep track of the structure of the stimulus. As more information becomes available from the array, descriptions are added to the object-tokens. The visual array can be accessed only via object-tokens. In the present experiments, it may be that on some trials perhaps only two object-tokens were created, one for each line of 
circles. If one of the object-tokens contained vertical and horizontal line feature information, then a plus could have been perceived.

Treisman and Gelade (1980) have proposed that feature integration is mediated by focal attention. They have proposed that features that fall within focal attention are likely to form conjunctions, features that fall outside of focal attention are likely to form conjunctions, but that features within focal attention are not likely to form conjunctions with features from outside focal attention. This theory could explain the present results if the allocation of attention is affected by some preattentive unit formation processes, as proposed by Kahneman (Kahneman, 1973; cf. Treisman, Note 1). Specifically, if attention is allocated to a particular perceptual unit or group, then features within that unit are likely to be integrated, features outside that unit are likely to be integrated, but features from that unit and another are not likely to be integrated. According to the view put forward here, perceptual organization would affect feature integration indirectly by its effect on the allocation of attention. In the present experiments, on some trials subjects may have attended to one line of circles or the other. If the vertical and horizontal line features both fell within (or without) focal attention, an illusory plus could have been perceived.

Wolford's (1975) feature perturbation theory could be modified, I believe, to describe the present results. According to Wolford, features are abstracted and are represented in a psychological space that is isomorphic in distance relationships to physical space. Features perturbate in this psychological space according to a random walk process. Features that are close together in physical space will be close together in psychological space and therefore more likely to perturbate to overlapping positions in this psychological space. This theory could be modified, I believe, to account for the present results. Distances in psychological space might not be affected only by physical distances, but also by such things as similarity, colinearity, etc. Features from two stimulus items that are physically far apart might be represented as being close together in psychological space if they are part of the same perceptual group. It is interesting to note that Coren and Girgus (1980) have found that perceptual organization affects distance judgments. Subjects judge distances between dots within a perceptual group as being smaller than identical physical distances between dots in different perceptual groups. In the present experiments, perhaps the psychological distance between items in the same line of circles is less than the psychological distance between items in different lines of circles. If features perturbated in this psychological space according to a random walk process, then features from items within a group would be more likely to overlap than would features from items in different groups.
The perceptual group principle of feature integration may help explain other effects of perceptual organization. For example, Banks and Prinzmetal (1976; Prinzmetal \& Banks, 1977) found that subjects were faster at detecting a target item in a stimulus display if the target did not form a perceptual group with the distractor items. It may have been that when the target was grouped with the distractor items, conjunction errors between the target and distractor were possible. With these displays, subjects may have slowed their search to avoid making a conjunction error (cf. Treisman \& Gelade, 1980). It is impossible to know post hoc whether the stimuli used by Banks and Prinzmetal were "conjunction stimuli" or "feature stimuli." Future research will be necessary to determine whether perceptual organization affects feature abstractions as well as feature integration.

Two approaches have emerged in the study of visual information processing. On one hand, feature analytic theories provide an explicit mechanism for recognition. On the other hand, many investigators have criticized feature analytic theories because they fail to provide an explanation for important configurational effects (Banks \& Prinzmetal, 1976; Mermelstein et al., 1979; Pomerantz et al., 1977; Weisstein \& Harris, 1974). The concern for an explanation of configurational effects has not, however, lead to an explicit theory of recognition. The perceptual group principle provides a functional link between these two approaches to the study of perception that may help explain some of the effects of perceptual organization while increasing our understanding of feature integration.

\section{REFERENCE NOTE}

1. Treisman, A. Perceptual grouping and attention in visual search for features and for objects. Manuscript in preparation, 1981.

\section{REFERENCES}

Banks, W. P., \& Prinzmetal, W. Configural effects in visual information processing. Perception \& Psychophysics, 1976, 19, 361-367.

BJork, E. L., \& Murray, J. T. On the nature of input channels in visual processing. Psychological Review, 1977, 84, 472-484.

Coren, S., \& Girgus, J. S. Principles of perceptual organization and spatial distortion: The Gestalt illusions. Journal of Experimental Psychology: Human Perception and Performance, 1980, 6, 404-412.

Fox, J. Continuity, concealment and visual attention. In G. Underwood (Ed.), Strategies of information processing. New York: Academic Press, 1978.

GaRner, W. R. The processing of information and structure. New York: Wiley, 1974.

Gibson, E. J. Principles of perceptual learning and development. New York: Appleton, 1969.

Kahneman, D. Attention and effort. Englewood Cliffs, N.J: Prentice-Hall, 1973.

Marr, D. Early processing of visual information. Philosophical Transactions of the Royal Society Bulletin, 1976, 275, 483-524. Mermelstein, R., Banks, W., \& Prinzmetal, W. Figural 
goodness effects in perception and memory. Perception \& Psychophysics, 1979, 26, 472-480.

Pomerantz, J. R., Sager, L. C., \& Stoever, R. J. Perceptions of wholes and their component parts: Some configural superiority effects. Journal of Experimental Psychology: Human Perception and Performance, 1977, 3, 422-435.

Prinzmetal, W., \& Banks, W. P. Good continuation affects visual detection. Perception \& Psychophysics, 1977, 21, 389-395.

Rumelhart, D. E., \& Siples, P. Process of recognizing tachistoscopically presented words. Psychological Review, 1974, 81, 99-118.

Santee, J. L., \& Egeth, H. E. Interference in letter identification: A test of feature-specific inhibition. Perception \& Psychophysics, 1980, 27, 321-330.

Selfridge, O. G. Pandemonium: A paradigm for learning. In The mechanisation of thought processes. London: H. M. Stationery Office, 1959.

SNYDE R, C. R. Selection, inspection, and naming in visual search. Journal of Experimental Psychology, 1972, 92, 428-431.

Treisman, A., \& Gelade, G. A feature integration theory of attention. Cognitive Psychology, 1980, 12, 97-136.

Treisman, A., \& Schmidt, H. Illusory conjunctions. Cognitive Psychology, 1981, in press.

Treisman, A., Sykes, M., \& Gelade, G. Selective attention and stimulus integration. In S. Dornic (Ed.), Attention and performance. Potomac, Md: Erlbaum, 1977.
Weisstein, N., \& Harris, C. S. Visual detection of line segments: An object superiority effect. Science, 1974, 186, 752-755.

Wolford, G. Perturbation model for letter identification. Psychological Review, 1975, 82, 184-199.

Wolford, G., \& Shum, K. H. Evidence for feature perturbations. Perception \& Psychophysics, 1980, 27, 409-420.

\section{NOTE}

1. The problem with applying signal detection theory in this research is that it is impossible to get a measure of detectability for same vs. different group stimuli that is independent for feature and conjunction displays. This problem arises because there are two different hit rates (same vs. different group), but four different false-alarm rates (same vs. different group and feature vs. conjunction). In a more fundamental sense, signal detection theory describes the discrimination of noise from an external signal. In these experiments, there is noise, external signals, and internal signals (illusory pluses).

(Manuscript received February 25, 1981; revision accepted for publication on June 29,1981 .) 